\title{
Das Apokryph „Unser lieben Frauen Traum“ bei den Kroaten im 18. Jahrhundert
}

\author{
ANDREA SAPUNAR \\ Staroslavenski zavod Hrvatskog filološkog instituta, Demetrova 11, HR-41000 Zagreb \\ E-mail: staslav@filolog.hfi.hr
}

\begin{abstract}
In a wider context, the filological analysis of valuable literary records shows that the apocryphal texts of the Croats from Burgenland are based on German examples and their roots go all the way back to the Middle Ages.
\end{abstract}

Die Gestalt der Gottesmutter steht der Sensibilität des mittelalterlichen Menschen sehr nahe und ist ihm sehr lieb. Daher findet man sie als Hauptfigur der verschiedenartigsten mittelalterlichen Literaturformen, deren Anwesenheit und Dauer oft sogar bis ins 19. Jh. reichen. Die der Marienthematik gewidmeten Werke sind zahlreich: von Gebeten, homiletischen Texten und Mysterienspielen bis zu Apokryphen und Versen. Die bekanntesten Apokryphen mit der Gestalt der Gottesmutter in der kroatischen Literatur sind: Uspenije Bogorodice (der Tod Mariens und ihre Himmelfahrt), Hođenje Bogorodice po mukama (Marias Herabsteigen in die Hölle sowie ihre Bitte um die Erleichterung der Qualen für die Sünder), Jakovljevo protoevanđelje (erzählt detaillierter von Marias Leben und Jesu Geburt) sowie San Divice Marije über die künftigen Leiden Jesu. Voller Ahnungen und Angst enthüllt Maria dem Sohn ihren schmerzlichen Traum, wobei sie dadurch die Prophezeiung seiner baldigen Leiden und der Passion ausdrückt. Am Ende des Apokryphen wird jenen, die an diese Ankündigung von Jesu Tod glauben, sie lesen, beten, abschreiben oder auf eine andere Art und Weise weiterverbreiten, Glück und Heil, Schutz vor dem jähen Tode und vor Krankheit versprochen, weiters das Himmelreich nach dem Tode usw.

Das Motiv vom prophetischen Traum Mariens und der Vision der Passio Christi ist in apokryphen Texten aus dem weiten Gebiet Süd-, Mittel-, West- und besonders Osteuropas vertreten, wo solche Texte sowie zahlreiche Versionen davon in Volksmeinung und -glauben eine deutliche Spur hinterlassen haben. Auf der Suche nach der Herkunft des alten Legendenmotivs berücksichtigt Mathilde Hain $^{1}$ zahlreiche Varianten, die in ganz Europa entstanden sind; von irischen, französischen, spanischen und italienischen Quellen bis zu ihnen ähnlichen Redaktionen in russischer, polnischer und anderen slawischen Sprachen. Ihrer Meinung nach tritt das Apokryph Unser lieben Frauen Traum als ein Ausdruck der mystischen Frömmigkeit vor, die mit Bernhard von Clairveaux (12. Jh.)

${ }^{1}$ Mathilde HaIn, Der Traum Mariens. Ein Beitrag zu einem europäischen Thema. Dona Ethnologica, Beiträge zur vergleichenden Volkskunde (Leopold Kretzenbacher zum 60. Geburtstag), Südosteuropäische Arbeiten, 71. München 1973, 218-232. 
beginnt und in der Passionsmystik des Franz von Assisi (13. Jh.) kulminiert. Die meditative Versenkung in das Leiden Christi bezieht auch Mariens Leiden mit ein; im 13./14. Jh. ist diese religiöse Bewegung noch ganz auf die klösterlichen Gemeinschaften beschränkt. ${ }^{2}$ Dort müssen wir auch den anonymen Verfasser des Marientraumes suchen; es war wahrscheinlich ein Mönch, der das sensible Herz Mariens die Passion des Sohnes vorausahnen ließ. ${ }^{3}$ Die Form des Traumes war keineswegs ohne Vorbild in der geistlichen Literatur; hingewiesen sei nur auf den altenglischen Traum vom Kreuze Christi (The dream of the road ${ }^{4}$ ) aus dem 10. Jh., wo aber die Gestalt Mariens fehlt. ${ }^{5}$

Das Motiv des Abschieds Jesu von seiner Mutter, das jahrhundertelang in der europäischen Tradition präsent ist, tritt erstmalig um 1280 in den Meditationes de Vita Christi des norditalienischen Franziskaners Johannes de Caulibus auf und wird in der geistlichen Literatur durch zahlreiche Handschriften, Übersetzungen und Abschriften weitertradiert. Im oberitalienischen Raume mochte laut M. Hains ${ }^{6}$ Überzeugung wahrscheinlich im 14. Jh. eine besondere Ausprägung des Apokryphs vom Marientraum der Heilandspassion entstanden sein. Sie dürfte in einer allerdings erst nachmittelalterlichen Ausbreitung aus dem romanischen Süden hinausschreitend im deutschen Sprachgebiet des späteren 16. Jh.s zu einer gewissen Verfestigung gekommen sein, wobei sich das Apokryph im katholischen Bereich weiter behauptete. ${ }^{7}$

Im Hinblick auf die Verbreitung des Apokryphs San Divice Marije im kroatischen Bereich sollte man seine Präsenz betonen sowie auch den mächtigen Einfluß auf die Grundstimmung in einem sehr ausgedehnten Raum, der sich vom nordöstlichen pannonischen Kroatien und heutigen Burgenland bis nach Istrien, zum Kroatischen Küstenland sowie entlang der dalmatinischen Küste bis in die südlichsten kroatischen Gegenden erstreckte. Die Verbreitung dieses apokryphen Textes im Laufe der Jahrhunderte bis in unsere Tage ist größtenteils, wie bei den meisten Völkern Südosteuropas, gerade durch die mündliche Weitergabe möglich geworden. ${ }^{8}$ Ebenso darf man auch die Rolle der gedruckten apokryphen Quellen in Form von Flugblättern oder Volksbüchlein nicht vergessen, die trotz

2 M. HAIN, a. a. O. 228.

${ }^{3}$ Ebenda.

${ }^{4}$ In: The Norton Anthology of English Literature, I. 1962; M. HAIN, a. a. O. 229.

${ }^{5}$ M. HAIN, a. a. O. 228.

${ }^{6}$ M. Hain stützt sich in ihrer Schlußfolgerung auf detaillierte Forschungen von Paolo TosCHI, Ancora sul «Sogno di Maria»: Lares 33 (Florenz 1967), 113-133; Il sogno di Maria: Rivista di Cultura Classica e Medioevale 2. (Rom 1965), 1104-1127.

7 Leopold KRETZENBACHER, Südost-Überlieferung zum apokryphen ,,Traum Mariens “: Bayerische Akademie der Wissenschaften, Philosophisch-historische Klasse (Sitzungsberichte, 1975/1). München 1975, 8.

${ }^{8}$ Die Texte apokrypher Gebete wurden größtenteils innerhalb der Familie mündlich weitergegeben, vom Vater auf den Sohn oder von der Mutter auf die Tochter. Ivo Franić, San Bl. Djevice Marije. Veoma raširen apokrif u našega naroda katoličke vjeroispovjesti: Vjesnik Etnografskog muzeja u Zagrebu, 1-2. Zagreb 1935, 110.

Studia Slavica Hung. 45, 2000 
kirchlicher Verbote oft auf Märkten neben Heiligenbildern und Ikonen verkauft ${ }^{9}$ und als beliebte Lektüre in großer Zahl unter dem Volk verbreitet wurden, das ihren Inhalt durstig aufsog und blind daran glaubte ${ }^{10}$.

Bezüglich der zahlreichen apokryphen Texte, die auf dem Boden des heutigen Kroatiens im 18. Jh. in Umlauf waren, und mit Sicherheit auch schon früher, kann man nur von Annahmen ausgehen, da wir keine ähnlichen gedruckten Exemplare aus älterer Zeit besitzen. ${ }^{11}$ Daher ist für uns als Bestätigung dieser Annahme ein Detail im Flugblatt von Girolamo Ostojić iz Staroga grada, od Isole Farsche na 2. Aprila $1813^{12}$ von großer Bedeutung, in dem der Koautor auf die Quelle seines Textes verweist, eine ältere Vorlage eines beliebten Volksgebetes aus dem Jahre 1760. Die ältesten heute bekannten kroatischen gedruckten Exemplare vom prophetischen Traum Mariens entstanden im Jahre 1780 auf dem Gebiet Westungarns und Niederösterreichs: Preblažene Divice Marie sanja und Molitva ku je jedan pobožni pustinac od svojega andjela čuvara prijel. ${ }^{13}$ Es handelt sich hierbei um kurze Prosatexte, die in der Form von kleinen Büchlein $(10 \times 8)$ gedruckt wurden. Außer dem Ausgabejahr sind uns alle übrigen Angaben bezüglich der Werke unbekannt: Sie sind anonym, ohne kirchliche Approbatur veröffentlicht, auch ohne Name des Druckers, der sie veröffentlichte. Blažene Divice Marie sanja erlebte noch eine spätere Ausgabe in einer neueren sprachlichen Bearbeitung, etwa gegen Ende des 19. Jh.s. ${ }^{14}$ Wir werden uns aber nun mit jenem Text beschäftigen, der in der zweiten Hälfte des 18. Jh.s entstanden ist.

Der Text der genannten apokryphen Werke wurde in Form von dünnen Volksbüchlein von acht und vierzehn Seiten herausgebracht, deren Titelblätter einfache Holzschnittillustrationen aufweisen. Die volksliterarische Mitteilung in Form von Volksbüchlein oder Flugblättern zählt der zeitgenössiche deutsche Wissenschafter Rudolf Schenda zu den sog. einfachen Formen der Volksliteratur, die auch durch die Möglichkeit der mündlichen Verbreitung des Inhalts

${ }^{9}$ I. FrANić, a. a. O. 102.

10 Ebenda.

11 D. ZEČEviĆ, Pučka književnost: Povijest hrvatske književnosti, Buch 1. Liber - Zagreb $1978,430$.

12 Das Flugblatt ist das sog. sveto oder Isusovo pismo, ein apokryphes Volksgebet, das laut Volksglauben in Jesu Grab gefunden wurde und in dem Jesus selbst seine Leiden beschreibt und am Ende Schutz vor dem jähen Tode, dem Satan, vor „Geistern“ im Haus sowie Frauen eine leichte Geburt bzw. jedermann Hilfe verspricht, der dieses Gebet trägt oder im Haus aufbewahrt. Thematisch ähnelt Jesu Brief dem Text des Marientraums. Daher kommen sie in Handschriften und gedruckten Büchlein sehr oft gemeinsam vor. Heute zählt dieses Exemplar aus dem Jahre $1813 \mathrm{zu}$ den Raritäten der National- und Universitätsbibliothek in Zagreb.

${ }^{13}$ Die Werke befinden sich heute in der Privatbibliothek von Stefan Zvonarich in Oberpullendorf.

14 Sanja Blažene Divice / MARIE. / U ZSELEZNI, / TISKA I NAKLADA EDUARDA DICK. L. KuZMICH, Kulturhistorische Aspekte der burgenlandkroatischen Druckwerke bis 1921 mit einer primären Bibliographie: Burgenländische Forschungen, Sonderband 10 (Eisenstadt 1992) $28 \mathrm{u}$. 226. Vgl. Nikolaus BENCSICS, Apokryphische Literatur bei den Burgenländischen Kroaten: Wiener Slavistisches Jahrbuch (Wien 1990), 13-19. 
charakterisiert ist. ${ }^{15}$ Die in solcher Form gedruckten Texte waren zum Tragen als Amulett gedacht und daher auch diesem Zwecke angepaßt, um denjenigen, der sie bei sich trug, vor jeglichem Übel und jeglicher Versuchung zu bewahren. ${ }^{16}$

Der apokryphe Text Preblažene Divice Marie sanja beginnt mit einer Erzählung als Hauptform der Deutung des Fabelstoffes, und zwar mit einer Erzählung in medias res, so daß schon im ersten Satz eine Angabe über die Gestalt und den Raum gemacht wird: Kada bi Divica Maria va Betlehemu, na brigu Oliveti zaspala. Danach folgt die Erzählung der Ereignisse: Doide k-njoj, nje dragi sinak, i reče njoj, predraga majka spiš, ali virostuješ, i odgovori ona, sam zaspala, ti si me prebudio. Strašno sam od tebe sanjala. Kod da bi ti bil va verti ulovljen, suši svezan pred Kajfaša peljan, od Kajfaša k-pilatušu od pilata kHerodešu, da su tvoju S. glavu zbili, sternjem korunili, iz sudske hiže van peljali, na tvoja $S$. ramena križ navalili, iz varoša na brig peljali, onde na križ prebili, tako visoko da te nisam mogla doseći tvoj bok preboden, da je kerv i voda van curila, i mene poškropila, potom su te S. križa doli z-neli va moje ruke kot mertva dali, ter zakopali, tako da je moje serce od žalosti puklo. Im Anfangsteil des Textes entfaltet sich die Erzählung der Ereignisse durch den allwissenden Erzähler, um bald von objektiver Narration in subjektive überzugehen bzw. in die direkte Rede in erster Person, was sicherlich als eindrucksvolles und sehr wirkungsvolles Mittel zur Verstärkung der Glaubwürdigkeit des Erzählten gewählt wurde. Durch die Wiederholung einzelner Wörter im Text wird der Inhalt betont und hervorgehoben, was auf den Leser/Zuhörer expressiv wirkt. Im folgenden Beispiel: ... suši svezan pred Kajfaša peljan, od Kajfaša k-pilatušu od pilata k-Herodešu ..., wird außer der Wortwiederholung auch eine Steigerung verwendet, durch die der Inhalt auf dem Wege zu einer Kulmination der Erzählung verstärkt wird, zu der man wiederum in dynamischem Rhythmus gelangt, der durch die asyndetische Verbindung des Satzes ermöglicht wird. Durch eine derartige Verbindung wird ein Rhythmus erzielt, der gerade im Kulminationspunkt den dramatischen Textausdruck formt.

Weiters kündigt Jesus die Deutung von Marias Traum als Wahrheit an, die sich tatsächlich ereignen wird und fügt hinzu: ... ki bude na tu sanju mislil, ali pri sebi nosil, on će od zloga očuvan biti, i neće z-naglum smertćum umriti prez sveti sakramentov. Ja i ti majka ćemo pri njegovoj poslidnjoj uri biti, i njegovu dušu va nebo zapeljati. Amen.

Danach unterbricht der Erzähler die Erzählung, um von der Wahrhaftigkeit

15 Divna ZeČEviĆ, a. a. O. 435; Rudolf Schenda, Volk ohne Buch. Studien zur Sozialgeschichte der populären Lesestoffe, 1770-1910. Frankfurt am Main 1970.

16 Ivo Franić veröffentlichte einige Texte des Traums der Jungfrau Maria mit der Anmerkung, daß man solche und ähnliche Texte fast in jedem Bauernhaus finden kann, gewöhnlich ,hinter den Trambaum in der Stube“ (za gredom u sobi) gesteckt. Die Frauen trügen dieses Apokryph in ihren Gebetbüchern, die Soldaten im Kriege eingenäht in ihre Blusen und Mäntel, daß sie dadurch „,bewahrt bleiben vor der Kugel und dem jähen Tode“. Wörtlich fährt Franić fort: „An seiner (des Apokryphs) Wunderkraft zweifeln die älteren Bauern nicht im mindesten. Wer dennoch daran Zweifel hegt, der versündigt sich und das Vertrauen erlischt.“'I. Franić, a. a. O. 103. 
und Nützlichkeit des folgenden Gebetes zu überzeugen. So wie bei anderen südosteuropäischen Texten, vor allem aber auch entsprechend mittel- und westeuropäischen Segensformeln des 12. Jh.s im Mittellatein und ab dem 14. Jh. auch deutschsprachig, findet man auch hier, wahrscheinlich unter dem Einfluß der mitteleuropäischen Texte, ${ }^{17}$ einen authentischen Beweis der „Nützlichkeit“ des Gebetes: Dieses Gebet schickte Papst $\mathrm{Leo}^{18}$ seinem Bruder, um ihn vor dem Feind zu bewahren. Leo selber hatte schon die Verheißung der Unverwundbarkeit durch Säbel oder Gewehr und den Schutz vor dem jähen Tode, dazu die Gewißheit, daß die guten Werke erhört werden. ${ }^{19}$ So wird auch jenen, die den Text dieses Gebetes sprechen, der Schutz vor dem jähen Tod versprochen, und niti voda niti oganj niti nepretelj neće moći njemu naškoditi. Noch kann das Feuer dem Haus, in dem dieses Gebet gesprochen wird, etwas anhaben. Durch die Wiederholung im erwähnten Teil des Satzes bzw. durch den betonten Inhalt wird die Wahrhaftigkeit der Behauptungen des Autors bekräftigt.

Es folgt ein kurzes abschließendes Gebet, als unerläßliche Frömmigkeitsformel in den apokryphen Werken, die nach fast fertigen, starren Mustern der Anrufung Mariens und Christi gearbeitet sind: Sveta Maria svojum pomoćum budi pri meni, Kristuš zveliči me, i občuvaj me od svega zla suprotivnoga, križ Kristušev pomozi meni, križ Kristušev obladaj sve moje nepretelje, križ Kristušev budi moj branitelj pred i za manum, Bog Otac, Bog Sin, Bog Duh Sveti, jedan jedini Bog od veka ča do veka. Amen.

Der zweite apokryphe Text Molitva ku je jedan pobožni pustinac od svojega andjela čuvara prijel wird am Anfang durch eine Formel der direkten Hinwendung zum Leser/Zuhörer strukturiert, was im allgemeinen die Anfänge vieler Werke der älteren Erzählprosa charakterisiert. Gerade solche bereits feststehende Formeln oder Topoi übernahm das Mittelalter aus der antiken Rhetorik und modifizierte sie in seinem Geist. ${ }^{20}$ Durch diese Hinwendungsformel versucht man die Aufmerksamkeit des Lesers/Zuhörers zu erhalten und ihn so in den Erzählkreis hineinzuziehen: Pobožni i ponizni keršćeniki, prosim vas va jime Jezu$\check{s}$, posluhnite na ovu skrovnu molitvu ku je jedan pustinac od svojega andjela čuvara prijel ... Die Erwähnung von Jesu Namen (va jime Jezuša) kann man als Berufung des Autors auf eine Autorität verstehen, was im Weltverständnis des mittelalterlichen Menschen immer von entscheidender Bedeutung war.

Befassen wir uns nun etwas näher mit dem Ausdruck: jedan pobožni pustinac. Die Gestalt des Einsiedlers und das Motiv des Einsiedlerlebens treffen wir oft in der mittelalterlichen Erzählprosa an, da dieses Motiv das Ideal des mittelalterlichen Lebens ausdrückt: die Entsagung von allem Irdischen und die Selbstaufopferung für den Glauben. Daher ist die Gestalt des Einsiedlers auch

17 L. KretZenbacher, a. a. O. 87.

${ }^{18}$ Gemeint sind doch wohl Papst Leo III. (795-816) und Karl der Große (768-814).

${ }^{19}$ L. KRETZENBACHER, a. a. O. 88.

20 Vgl. E. R. Curtius, Europäische Literatur und lateinisches Mittelalter. Bern und München ${ }^{3} 1961,89-116$; D. FALIŠEVAC, Hrvatska srednjovjekovna proza. Zagreb 1980, 105. 
später in der barocken Literatur besonders beliebt. ${ }^{21}$ Die Wendung jedan pustinac steht an der Stelle des Vor- und Zunamens der Teilnehmer an den Ereignissen und bezeichnet auf diese Art und Weise in erster Linie seine Stellung in der Gesellschaftshierarchie, gerade des Strebens der Volkserzählung nach der Glaubwürdigkeit der erzählten Ereignisse wegen. „Solche namenlose Gestalten konnten einen Dienst erfüllen und boten auch die offene Möglichkeit einer breiten Mitteilung, einer Abstraktion, was die Rückkehr zu den Grundwerten bedeutet, denen die einzelne Figur als gestaltloser, d.h. nicht indvidualisierter Träger dient. [...] Durch die Bezeichnungen neki, neka, jedan wird ein Ereignis aus dem alltäglichen Leben herausgenommen und die Gestalt des Protagonisten betritt dadurch die literarische Organisation, deren Maßstab die Konzeptordnung ist, die Ordnung jener Werte, die potentiell für alle Angehörigen einer Gemeinschaft gelten." 22

Ein frommer Einsiedler erhielt ein Gebet von seinem Schutzengel, $i$ kada bi zišnjim na umiranje došlo, tako je on ovu molitvu svetomu Clementi papi oćitoval, $i$ obećal. Wie es bei apokryphen Texten oft der Fall ist, beruft sich der Autor auf eine bereits bekannte Autorität, in diesem Fall auf Papst Clemens, der ihm als zuverlässiger Garant der Wahrheit dienen soll, die er mit folgenden Worten ausdrücken möchte: ... da ki goder človik bude pri sebi nosil (ovu molitvu) od njega moraju svih zli duhi odstupiti, noći $i$ va dne, $i$ va koj hiži je ova molitva ne more oganj ali oblisk strelje naškodit, žena ka teško dite porađa, neka postavi ovu molitvicu na persih ali na glavu svoju tako će prez velike muke dite poroditi.

Wie in den Mysterienspielen, besonders des 17. und 18. Jh.s, in denen wir zahlreiche Wahrhaftigkeitsbeweise in den verschiedensten Varianten vorfinden, ist auch hier das bekannte probatum est präsent bzw. der Beweis, daß das Gebet „erprobt" ist. Der objektive und allwissende Erzähler, der über alles, was sich ereignet hat, gut informiert ist, berichtet uns: Skusilo se je jednuč va varoši pragi pri jednoj ženi, ka je jur petero dice mertve porodila. Kad bi pak šestim noseća bila, i da bi je rodila, postavila je ovu molitvu na glavu svoju, taki je živo dite zveseljem porodila. Skusilo se je još jednuč pri muškoj glavi ki je osam let, tri sto zali duhi obseden bil, zel je jedan mašnik iz tovaruštva Jezuša ovu molitvu i pred njim je nju zmolil, i njemu na glavu postavil, taki i jesu iz njega zli duhi van prošli. Die Namen der Personen sind kompensiert und so tragen die namenlosen Gestalten einer Frau oder eines Priesters als typisches Produkt volkstümlicher Literaturschöpfungen zur Wahrhaftigkeit der Ereignisse bei. Im Laufe der Erzählung gibt es häufig einen Wechsel von Polysyndeta und Asyndeta. Dadurch entsteht auch der doppelte Rhythmus, der den Rhythmus der Erzählung belebt.

Weiters werden noch ein Gedanke und ein Versprechen hinzugefügt, was immer von Vorteil ist, wenn man im von Angst erfüllten Menschen Vertrauen und festen Glauben wecken möchte, nämlich: Ki človik bude pri sebi nosil,

${ }^{21}$ D. FALIŠEVAC, a. a. O. 63.

22 D. ZEČEVIĆ, a. a. O. 431.

Studia Slavica Hung. 45, 2000 
onome će Kristuš tri dni pred njegovum smerti oznanit, ki pak ju bude molil sedam petkov spolu, i ofruje za svoje pokojne pretelje ali rođake, ali za jednu ubogu purgatoriumsku dušicu tako će oslobojena biti ... Und danach weiter: ... va koj hiži si bude ova molitva, neće kuga ali velika nevolja ladati zato neka ne bude nedan človik len nju većkrat moliti, ali pri sebi nositi. Die einzelne Anmerkung bezieht sich auf die analphabetischen Gläubigen: Ki pak ne zna štat, ta nek z-moli po petke sedam Otac naš i sedam Zdravi M. i Veru božju ter hvalu ljute muke Kristuševe. Der Gebetstext selbst beginnt mit der Anrufung Jesu, die dann wie ein Leitmotiv wiederholt wird, um immer wieder eine neue Gedankeneinheit zu eröffnen. Die Wiederholungsfigur hat hier nicht nur die Funktion der Betonung und Hervorhebung des Erwähnten, sondern wird durch ihre rhythmische Wiederholung zum Strukturierungsprinzip der Textkomposition. Durch die Wiederholung der Ausdrücke Jezuš, ti si ... und 0 , moj Jezuš ... erhält der Stoff eine musikalische Komposition. Diese Wiederholungsfunktion ist ebenfalls von der Kategorie des mündlichen Hersagens bestimmt, des Hersagens in einem geschlossenen Erzählkreis. ${ }^{23}$

Der Text des Gebets lautet: Predragi Jezuše Kristuše ja preštimavam tebe tvoje sv. človičanstvo ko si z-voljum otca Boga, i od duha svetoga va utrobu Blažene Divice Marije prijet i rođen ... o Jezuš ti si svoju svetu kerv sterpljeno za nas grišnike i grišnice proljal, o Jezuš ti si nam kroz tvoju žuku muku i smert nebeska vrata odperl, o Jezuš ti si veliko uboštvo i progonstvo od nepreteljov tri trideset let sterpljeno za nas grišnike preterpil, o moj Jezuš ja premišljavam tvoje tužno razlučenje od predrage Majke Marije. O moj Jezuš ja mislim na tvoju pokornu molitvu na Brigu oliveti da si od mlavosti kervavi pot točil prik tvojega sv. obraza o moj Jezuš ja premišljavam kako si ulovljen sužem svezan od jednoga sudca do drugoga peljan bil, i tvoje sveto telo z-biči razdrapano da je tvoja sveta kerv, prig tvojega tela curila, $i$ onda su ti oštru ternovu korunu na tvoju sv. glavu pritisnuli, da je jedan oštri tern tvoj sveti verhunac ..., i tako va sv. ... tvoji ostal, o moj Kristuš ja preštimavam, kako si ti z-jednim teškim križem obložen bil i moral si sam na brdo Kalvariju nositi, da si duboke rane na tvoji sv. ramena dostal. O moj Jezuš ja mislim, kako si ti gol na sv. Križ pribit bil, o Jezuš ti si tri ure na sv. Križu živ ostal, jesi sedam moćni riči sprogovoril, po ... o moj Jezuš iz sv. ... doli znet bil. O moj Jezuš z-tvojum presvetum žukum mukum $i$ smertjum, i s timi svetimi sedmini ričami, ću ja N.N. moje telo i dušu na veke kripiti. Amen. ${ }^{24}$

Die Sprache der Apokryphen Preblažene Divice Marie sanja und Molitva ku je jedan pobožni pustinac od svojega andjela čuvara prijel ist sehr einfach, volkstümlich und leicht verständlich, was allgemein ein Vorzug der apokryphen Literatur ist, die für ein möglichst breites Leserpublikum geschrieben ist. Die Rechtschreibregeln werden sehr inkonsequent befolgt.

${ }^{23}$ D. FALIŠEVAC, a. a. O. 130.

${ }^{24}$ Einzelne Textteile sind in ziemlich schlechtem Zustand, so daß es leider nicht möglich war, den Text zur Gänze anzuführen. 
Weiters stellt sich uns die Frage der Quellen bzw. der Vorlagen, nach denen diese burgenländischkroatischen Texte entstanden sind, denn die apokryphen Texte wurden, wie bereits bekannt ist, in zahlreichen Handschriften, Übersetzungen und Abschriften verbreitet. Da wir keine kroatischen gedruckten Exemplare des Marientraums des 17. und 18. Jh.s oder aus noch älterer Zeit aus der alten Heimat besitzen, wenden wir uns der Erforschung anderer alter apokrypher Quellen zu, in erster Linie in deutscher Sprache.

Die Sammlung abergläubischer Gebete und Segnungen Geistlicher Schild 25 war im 17. und 18. Jh. ein weit verbreitetes Volksbuch, was seine zahlreichen Ausgaben, angefangen von den ältesten in Mainz 1613 und 1647 bis zu den letzten Ende des 19. Jh.s, beweisen. ${ }^{26}$ Die einzelnen Teile dieser Kompilation reichen noch viel tiefer in die Vergangenheit zurück, was vor allem auf die Offenbarungen und Gebete der hl. Birgitte, der königlichen schwedischen Heiligen (1303-1373), gilt. Neben den Texten der hl. Birgitte findet sich bis in die Zeit der letzten Ausgaben der Sammlung Geistlicher Schild im 19. Jhr. fast regelmäßig ein kurzer Dialog zwischen Jesus und Maria, bekannt unter dem Titel Unserer lieben Frauen Traum. Das Thema beider apokrypher Texte ist die Passio Christi: Die hl. Birgitta erfährt in ihren Visionen aus Mariens Mund von Christi Leiden, während die Mutter im San divice Marije alle Leiden und Schmerzen ihres Sohnes vorhersieht und ahnt. Daher sind diese beiden Texte in den Gebetbüchern des 18. Jh.s oft gemeinsam zu finden. Während wir nun aber, was den Text der hl. Birgitte anbelangt, genau die Herkunft, die Art und Weise sowie die Wege der Ausbreitung bestimmen können, verlieren sich die Spuren des Apokryphs vom Marientraum in der Zeit vor der Sammlung Geistlicher Schild aus dem Jahre 1613 im deutschen Sprachgebiet, auch wenn man heute annimmt, daß das Gebet schon aus früherer Zeit stammt und seine Wurzeln weit ins Mittelalter zurückreichen.

Wenden wir uns nun dem kroatischen Text San Blažene Divice Marije zu, der $1780 \mathrm{im}$ Gebiet des heutigen Westungarns und Niederösterreichs entstanden ist. Bei einer Vergleichsanalyse zwischen dem burgenländischkroatischen Text und dem deutschen Apokryph Unser lieben Frauen Traum aus der Sammlung Geistlicher Schild, 1647 in Mainz gedruckt, bemerkt man eine ganze Reihe von Übereinstimmungen. Die Erzählung in den beiden Apokryphen verläuft sogar fast identisch; die Abweichungen hingegen sind eher unbedeutend. Einzelne burgenländischkroatische sprachliche Ausdrücke und Satzkonstruktionen weisen

25 „Geistlicher Schild gegen geist- und leibliche Gefährlichkeiten allzeit bey sich zu tragen. Darinn sehr kräftige Segen und Gebethe so theils von Gott offenbaret, von der Kirchen H.H. Vätern gemacht, und von Urbano VIII. Röm. Papst approbiert worden. Zum Trost aller Christgläubigen, sonderlich deren, so zu Wasser oder Land reisen, damit sie durch Kraft dieses bey sich tragenden Schilds, vor vielen Gefahren erhalten werden. - Cum Licentia Ord. Cens. Trevir. ibidem An. 1647 impressum. Gedruckt zu Maynz.“

${ }^{26}$ M. HAIN, a. a. O. 218.

Studia Slavica Hung. 45, 2000 
ebenfalls auf eine wörtliche Übersetzung der deutschen Vorlage hin. Zum Vergleich führen wir beide Texte an:

\begin{tabular}{|c|c|}
\hline Sanja Blažene D. Marie & Unser lieben Frauen Traum \\
\hline Jezuš Kristuš pravi Božji Sin $i$ čiste & Jesus Christus Gottes Sohn und reiner Jungfrauen \\
\hline Divice Marie. Kada bi Divica Maria va & Mariä. Als die heilige Jungfrau Maria zu Bethlehem \\
\hline Bethlehemu, na brigu Oliveti zaspala. & auf dem Berg eingeschlaffen war; Kam zu ihr der \\
\hline Doide k-njoj, nje dragi sinak, $i$ reče & Sohn Gottes, ihr lieber Engel, und sprach zu ihr: \\
\hline njoj, predragc & t du? \\
\hline tuješ, i odgovori ona, sam zaspala, ti si & Sie sprach: Ich hab geschlaffen, und $d u$ hast mich \\
\hline $\begin{array}{l}\text { me prebudio. Strasno sam od tebe } \\
\text { sanjala. Kod da bi ti bil va verti ulov- }\end{array}$ & $\begin{array}{l}\text { erweckt, und hat mir erschrocklich getraumet; Ich } \\
\text { habe gesehen, als wärest du im Garten gefangen, mit }\end{array}$ \\
\hline ljen, suži svezan pred Kajfaša peljan, & Stricken gebunden, von Caipha zu Pilato, von Pilato \\
\hline od Kajfaša & zu Herode \\
\hline rodešu, das & Haupt geschlagen, mit Dornen gecrönet, und aus dem \\
\hline $\begin{array}{l}\text { njem korunili, iz sudske hiže van } \\
\text { na tvoja S. ramena križ nave }\end{array}$ & $\begin{array}{l}\text { Richt-Haus geführet, Holtz auf deine heilige Achsel } \\
\text { gelegt, aus der Stadt auf den hohen Berg geführet, }\end{array}$ \\
\hline varoša na brig pel & und ans Creutz geschlagen haben, so hoch, daß ich \\
\hline prebili, tako visoko da te nisam mogla & dich nicht habe erreichen können. Deine heilige Seite \\
\hline doseći tvoj bok preboden, da je kerv $i$ & durchstochen, daraus Blut und Wasser geflossen, und \\
\hline voda van $\mathrm{cu}$ & $\begin{array}{l}\text { auf mich getröpflet hat } \\
\text { und also gecreutzigt in }\end{array}$ \\
\hline $\begin{array}{l}\text { tom su te } S . \text { kriza doli z-neli va moje } \\
\text { ruke kot mertva dali, ter zakopali, tako }\end{array}$ & $\begin{array}{l}\text { und also gecreutzigt in meine Hände wie einen Todten } \\
\text { gelegt und begraben, so daß mir aus grossen Schmer- }\end{array}$ \\
\hline da je moje serce od žalosti puklo. Reče & tzen das Herz hätte zerspringen mögen. Jesus sprach \\
\hline Jezuš njoj, draga maj & zu ihr: Meine allerliebste Mutter, es ist dir ein wahr- \\
\hline jistinska sanja & hafter Traum fürkommen. Wer diesen Traum gedenken \\
\hline sanju mislil, & agen wird, der wird von allen bösen \\
\hline zloga očuval biti, i $n$ & yn, und wird nit jähling sterben, \\
\hline $\begin{array}{l}\text { cum umritt prez svetl sakramentov. Ja } l \\
\text { ti majka ćemo pri njegovoj poslidnjoj }\end{array}$ & $\begin{array}{l}\text { auch nicht ohne Empfahung des Helligen Sacraments } \\
\text { aus dieser Welt verscheiden, wir werden bey seinem }\end{array}$ \\
\hline $\begin{array}{l}\text { uri biti, i njegovu dušu va nebo za- } \\
\text { peljati. Amen. }\end{array}$ & $\begin{array}{l}\text { letzten Ende seyn, und seine Seel in das Himmelreich } \\
\text { einführen. }\end{array}$ \\
\hline
\end{tabular}

Als sehr wahrscheinlich erscheint die Tatsache, daß der burgenländischkroatische Text gerade durch die Übersetzung dieser deutschen Vorlage aus der Sammlung Geistlicher Schild selbst oder einer ihrer Abschriften in einer Handschriftensammlung von Gebeten des 18. Jh.s entstanden ist, wie dies z.B. das Geistliche Vergißmeinnicht (1771) und die Katholischen Gebethe für die Jugend und Alter (1785) sind. ${ }^{27}$ Neben dem Abdruck in der Sammlung Geistlicher Schild und handschriftlichen Gebetbüchern begegnen wir dem San Divice Marije als eigenständigem Gebetsblatt oft im siebzehnten und 18. Jh. in zahlreichen Wallfahrtsorten des deutschen Sprachraums..$^{28}$

In solchen eigenständigen Blättern oder Volksbüchlein findet man gewöhnlich keine Hinweise auf den Drucker und das Ausgabejahr, meistens handelt es sich um Kleinformatausgaben auf billigem Papier mit einem primitiven Marien-

${ }^{27}$ Beide handschriftliche Gebetbücher werden heute im Österreichischen Museum für Volkskunde in Wien aufbewahrt. M. HAIN, a. a. O. 220.

${ }^{28}$ M. HAIN, a. a. O. 219. 
bild in Holzschnitt auf der Titelseite. ${ }^{29}$ Noch wahrscheinlicher wäre freilich die Möglichkeit, daß das burgenländischkroatische Exemplar des Marientraums gerade als Übersetzung eines solchen Gebetsblatts oder Volksbüchleins enstanden ist, und dann für die Bedürfnisse eines Wallfahrtsortes Westungarns oder Niederösterreichs, der häufig von Kroaten besucht wurde, gedruckt wurde. Neben dem apokryphen Flugblatt Unser lieben Frauen Traum findet man in Wallfahrtsorten um das Jahr 1750 verhältnismäßig oft auch ein Gebetsblatt über den Einsiedler. ${ }^{30}$ Ich nehme an, daß dieser Text seinem Inhalt nach dem burgenländischkroatischen Molitva ku je jedan pobožni pustinac od svojega andjela čuvara prijel (1780) entsprechen könnte.

Daher liegt der Schluß nahe, daß die angeführten Beispiele apokrypher Texte der burgenländischen Kroaten im 18. Jh. aufgrund deutscher Vorlagen entstanden sind und ihre Wurzeln aus diesem Grund tief ins Mittelalter reichen. Somit sind sie Teil einer langen Überlieferung apokrypher Texte in der europäischen Tradition.

29 Ebenda.

${ }^{30}$ Ebenda. 